\title{
Puntos de articulación entre violencia social, exclusión y violencia escolar
}

\author{
Intersections between social violence, exclusion \\ and violence in schools
}

Guadalupe Poujol Galván*

\begin{abstract}
RESUMEN
Del análisis de artículos, reportes de investigación y narrativa de docentes se desprende que algunos de los puntos que articulan la violencia social con la violencia escolar emergen de la situación de violencia desbordada por la que atraviesa México. Un tipo de guerra como el que se libra en el país, asegura para los poderes trasnacionales el saqueo de recursos y mercancías ilegales, con la complicidad del Estado, que deja avanzar la desigualdad, la exclusión, la impunidad. En la escuela, a la que acude una proporción importante de niñas, niños y jóvenes víctimas de la exclusión social, se reproducen las relaciones de uso de la fuerza, arbitrariedad e impunidad que se generan en la sociedad más amplia, quebrantando en los educandos el sentido de la educación como posibilidad de futuro.
\end{abstract}

Palabras clave: problematización, violencia social, violencia escolar, exclusión, educación básica

\section{ABSTRACT}

An analysis of articles, research reports, and teachers' narratives suggests that some of the points linking social violence with school violence are due to the exacerbated violence Mexico is experiencing. The type of war that is being waged in the country allows for the continued looting of resources and illicit goods on the part of transnational powers, with the complicity of the state. This in turn fosters further inequality, exclusion and impunity. At schools -which a significant proportion of the children and youth who are victims of social exclusion attend-, relationships characterized by the of use of force, arbitrariness and impunity generated in the larger society are reproduced, thus breaking in students the sense of education as a possibility for a better future.

Key words: problematization, social violence, school violence, exclusion, basic education

\section{INTRODUCCIÓN}

La preocupación de fondo de este estudio es acercarse a la comprensión del fenómeno de las distintas formas de violencia que se producen dentro de la escuela y las relaciones que estas tienen con la violencia social. Se trata de evitar en una visión reduccionista del problema que señala como violentos a niños, niñas y jóvenes, ocultando la complejidad del entramado en el que se genera la violencia.

\footnotetext{
“Universidad Pedagógica Nacional, Morelos, México; gpoujol@yahoo.com.mx
} 
Para dar cuenta de este entramado, se han considerado visiones geopolíticas e históricas, como la desarrollada por Ana Ceceña (2014), quien analiza el impacto de las fuerzas hegemónicas (Estados Unidos y sus aliados en el mundo) en Latinoamérica y el Caribe, en el siglo XXI. Estas fuerzas ejercen su dominio mediante una lógica de acumulación y concentración internacional inequitativa del capital.

Como estrategia de dominio instrumentan la guerra, que asegura el saqueo e impulsa negocios inmorales e ilícitos: armas, drogas, alimentos, trata de personas, mercenarismo, entre otros. Las condiciones de excepción implantadas favorecen un control no legitimado sobre las poblaciones, que configura, en palabras de Ceceña, un concepto de dominación de espectro completo que se alcanza mediante tres estrategias: avasallamiento, simultaneidad e impunidad. Avasallamiento en forma de tareas de prevención y disuasión, como una purga dirigida a las personas que estorban al saqueo. La simultaneidad se refiere a que se ataca al enemigo sin tregua, por todos lados y al mismo tiempo. Ceceńa (2014: 3) alude, en el caso mexicano, a aquella "en la que se aprobaron en cascada reformas [constitucionales] antipopulares (laboral, fiscal, de comunicaciones, educativa y energética...) que transformaron sustancialmente y de golpe la relaciones laborales, las pautas educativas, el patrimonio del pueblo de México, los niveles salariales y de imposición, la vigilancia o intromisión en la vida privada y los márgenes de maniobra de la sociedad".

La impunidad, según la misma autora, alude al vaciamiento de la justicia, a la ostentación de comportamientos ilegales, a la pérdida de referentes que garanticen el Estado de derecho; al contexto en el que aparecen simultáneamente el estado de excepción y la tierra de nadie.

La perspectiva de Ceceña se complementa con la del Equipo Bourbaki (2011: 11), que analizó el costo humano de la guerra en la construcción del monopolio del narcotráfico en México en el periodo 2008-2009. Estos investigadores citan a Elías Canetti para quien "una guerra es una guerra porque incluye muertos en el resultado".

Este equipo plantea que el uso de la fuerza por el control de los territorios de producción y distribución de las nuevas 
mercancías ilegales garantiza la acumulación capitalista ampliada, parte de un proceso global que trasciende su territorialidad. Por tratarse de mercancías todavía ilegales, se hace necesaria la utilización y protección del aparato del Estado, que da como resultado una interpenetración entre el "orden delictual" y el "orden legal”. Un hallazgo relevante de este equipo para la comprensión de la violencia en el país es que el costo humano de la guerra es una especie de "cuota" de exterminio selectivo que el Estado mexicano cobra a la sociedad, quien con sus acciones reivindica sus derechos humanos y los de la colectividad.

En este trabajo se estudia la violencia escolar en el entramado social de guerra descrito por Ceceña por considerar que en la escuela se expresan de diversas maneras el avasallamiento, la simultaneidad y la impunidad social y en ella gana fuerza, en palabras de Santos (2010), la lógica de la apropiación/violencia, en detrimento de la lógica de la regulación/emancipación que, según este autor, a nivel social está no solo contrayéndose, sino contaminándose internamente por la lógica de la apropiación/ violencia.

Se parte de considerar a la violencia como relación de poder, vínculo o forma de relación social por la cual uno de los términos ejerce su poder acumulado sobre el otro. Violencia es, pues, toda situación de poder que naturalice la desigualdad y la jerarquía, que transforme a una porción de sujetos en objetos (Maldonado, 2004: 5). La violencia objetiva se conjuga con la violencia simbólica planteada por Bourdieu (1999), para quien es esa que arranca sumisiones que ni siquiera se perciben como tales, apoyándose en expectativas colectivas y en creencias socialmente inculcadas.

Al estudiar la violencia, conviene abordarla desde cuatro elementos, los primeros tres propuestos por Moreno (2006): el acto (realización de poder acumulado sobre el otro, según Maldonado), los actores (agresor, individual o colectivo, que causa dańo objetivo o subjetivo a otra persona o grupo) y la significación de la acción (violencia cuando el agresor, el agredido o un observador de la acción dieran al acto excesivo una significación negativa para la víctima, siendo la intención de dańar el eje articulador de dicha acción); a estos tres elementos propuestos por Moreno se agrega el contexto como un campo de lógicas y fuerzas 
en el que se produce la violencia que pueden caracterizarse como de apropiación/violencia o de regulación/emancipación.

$\mathrm{Al}$ analizar el significado de la violencia en la historia, Madriaza (2006) plantea que la modernidad y el ejercicio de dominación han sustentado la violencia como un modo de sostener su propia simbolización y efectividad de predominio. Para este autor, la dirección que toman las investigaciones de la violencia escolar se basan en una definición que se fundamenta en la asimetría del ejercicio del poder y la fuerza. Madriaza advierte que es importante tener en cuenta que en la dinámica de la violencia escolar, más allá de la intimidación, hay un fondo social activo desde el cual emerge, que esta puede ser un síntoma, una forma que adquiere la tensión soterrada, para emerger en lo social. Una fuente de tensión mencionada por este autor es la violencia del sistema educativo.

\section{METOdOLOGÍA}

La estrategia teórico metodológica de la investigación documental y empírica se fue construyendo como un proceso de problematización (Zemelman, 1987), que implica la lógica de inclusión permanente de procesos, niveles y conceptos, que se conjugan en un momento histórico dado. Se trata de reconstruir la relación dinámica entre estos elementos, de forma que la reconstrucción articulada de la realidad social no simplifique su complejidad.

La perspectiva de la parte empírica de la investigación es holística, buscando relaciones en los datos que aportan los propios sujetos que, organizados en categorías, han permitido llegar a una reflexión teórica, de un modo interpretativo (Yurén, 2011).

La voz de los sujetos ha sido recuperada mediante la investigación narrativa. Al respecto, Blanco (2011) plantea que el eje fundamental es hacer sentido de la experiencia que, como toda herramienta de investigación social, requiere evidencias, plausibilidad interpretativa y un pensamiento disciplinado.

Se ha optado por el análisis paradigmático de contenido que, según Leiblich (1998), examina las similitudes y diferencias temáticas existentes entre diferentes relatos. El principal potencial de esta forma de análisis reside en su capacidad para desarrollar un conocimiento general sobre temas centrales que 
constituyen el contenido de las narraciones, en este caso, el contexto de la violencia escolar.

El análisis documental se ha organizado en dos apartados: en el primero, se presentan los datos sobre la exclusión social y la violencia escolar, las representaciones de diversos actores. Las fuentes de información son: la prensa, informes de organismos internacionales y revistas especializadas. Un segundo apartado en el que se revisan reportes de investigación educativa y sociológica que analizan las múltiples dimensiones del fenómeno de la violencia escolar.

En la investigación empírica se analizan algunos rasgos de los contextos comunitarios e institucionales en escuelas de educación básica en México a partir de la narración de cuatro profesores de primaria que enfrentan el problema de la violencia.

Los docentes narran los hallazgos que corresponden a una fase exploratoria, con base en observaciones y conversaciones con diferentes actores escolares, alumnos, docentes, padres de familia.

\section{RESULTADOS}

Las tres características de una guerra contemporánea planteadas por Ceceña: avasallamiento, simultaneidad e impunidad están presentes en nuestro país y operan activamente en la producción de la violencia y el despojo. La pobreza, la desigualdad y la exclusión están relacionadas con el avasallamiento, pues la población es tratada como una masa a la que no se le pone atención, se reducen sus posibilidades de bienestar y de gestionar sus derechos. La pobreza es una condición en la que se dan, de manera simultánea, la carencia, la inseguridad, la negación de futuro.

Para Kruijt (2008) los años de reestructuración económica y social, desde mediados de los ochenta y los noventa, transformaron no solamente el orden político, sino también la estructura económica y social de la región latinoamericana. El resultado global de estos cambios se refleja en una pobreza masiva, en una informalización de la economía y la sociedad y en un proceso de exclusión social de una gran parte de la población.

En el informe de la CEPAL (2013), México es el único país de América Latina donde creció la pobreza, por sus menores tasas de 
crecimiento económico y por el aumento de la desigualdad. El número de mexicanos que vive por debajo de la línea mínima de bienestar subió a 60.6 millones de personas, un millón más que en 2010.

Márquez (2014) caracteriza como bullying económico la pobreza en la infancia y la adolescencia, pues presentan mayores niveles de pobreza que el resto de la población mexicana. En 2010, $46.2 \%$ de la población era pobre, donde se incluye $53.8 \%$ de aquellos con 0 a 17 ańos. Colombo (2007) advierte que las sociedades desiguales condenan a sus poblaciones infantiles a condiciones insuficientes e inadecuadas de alimentación, sanidad y contención social. Estas condiciones configuran un daño social del cerebro infantil.

Las políticas públicas implementadas en el país para atenuar la pobreza extrema no han tenido el impacto esperado. Así lo reporta González (2014) quien, con datos de la CEPAL y la OIT, concluye que el impacto laboral del Programa Oportunidades es limitado y no ha mejorado la condición laboral de los beneficiarios, ya que $40.2 \%$ de mujeres y $74.1 \%$ de hombres se ubican en una categoría laboral igual o inferior a la de sus padres.

Si la violencia es toda relación de poder que naturaliza la desigualdad, más de la mitad de los mexicanos son víctimas de violencia estructural, de una estrategia de avasallamiento que los despoja de un territorio, de condiciones laborales y de vida dignas. Al nivel de la región latinoamericana, siguiendo a Kruijt (ibid.), una de las consecuencias de la pobreza y la exclusión social ha sido la gradual erosión de la legitimidad del orden civil, político y público. La economía y la sociedad informal se hallan excluidas del empleo estable, del ingreso regular, de los sindicatos, del acceso a las instituciones sociales que proveen servicios para satisfacer necesidades básicas como salud pública, vivienda popular, educación. La situación de formalidad-informalidad, legalidadilegalidad o criminalidad ha configurado una reestructuración del orden social en la región, dando lugar a sectores económicos paralelos, jerarquías sociales paralelas y estructuras institucionales paralelas, todo ello, caldo de cultivo para lo que este autor llama la violencia desbordada de la región.

En la situación de guerra, al avasallamiento, tomado como pobreza y abandono, se suman la simultaneidad de situaciones 
adversas en México, como un alto nivel de violaciones al derecho a la vida y la impunidad. El relator especial de la ONU sobre ejecuciones extrajudiciales, sumarias o arbitrarias, Christof Heyns, reportado por Camacho (2014), denunció que la vida está bajo seria amenaza en el país, en medio de una impunidad sistemática.

Garduño y Méndez (2009) citan a Irvin Waller, quien sostuvo que la descomposición de las estructuras sociales en México ha propiciado que el país se encuentre hoy en el quinto lugar mundial con altos índices de delincuencia organizada, y en decimotercero en delitos de orden común. Para Waller, tal incidencia obedece a las complicadas condiciones que enfrenta la juventud, pues vive en relativa pobreza, violencia familiar, padece exclusión o deserción escolar y desempleo, cultura de violencia, alcoholismo en su entorno y relativa facilidad para acceder a las armas.

En una situación social adversa de apropiación/violencia en la que aumenta la pobreza, el desempleo, la inseguridad y la impunidad, la violencia escolar se vuelve motivo de preocupación gubernamental y social.

Para Gabriela Rodríguez (2014), los golpes, agresiones, venganza y muerte son vida cotidiana entre muchos de los niños y nińas México y cita el caso de Angelina, adolescente indígena de 16 años agredida en su escuela. Angelina sufrió dos años de agresiones continuadas hasta culminar en una golpiza, grabada y exhibida en redes sociales. Ningún miembro de la comunidad educativa intervino para detener este caso.

Rodríguez analiza los resultados de la Consulta Infantil y Juvenil realizada por el IFE en 2012. De 2 millones 256532 niñas, niños y jóvenes entre 6 y 15 años de edad consultados, se encontró que casi una quinta parte vive maltrato en su casa, el cual disminuye conforme aumenta la edad. La violencia no ocurre solamente en la familia, hay también maltrato en las calles y en las escuelas, por parte de jóvenes, adultos y maestros.

El Estudio Internacional sobre la Enseñanza y el Aprendizaje 2013 (TALIS) de la OCDE, señala que casi 30\% de los maestros estima que hay intimidaciones. De acuerdo con el análisis comparativo de este estudio, México tiene el porcentaje más alto de 33 naciones participantes en número de docentes que reportan daño físico causado por la agresión entre alumnos, pues al menos 
una vez a la semana sus estudiantes lo enfrentan, casi cinco veces más que la media de 2.3, para el resto de los estados participantes.

En cuanto a las condiciones de alumnos y docentes, el estudio mencionado señala que casi la mitad de los profesores de secundaria labora en planteles con más de $30 \%$ de alumnos en condiciones económicas desfavorables, lo que duplica la media de los estados participantes. Los docentes destinan 24\% de su tiempo a tareas administrativas y a tratar de mantener el orden en las aulas.

Las políticas públicas con las que el gobierno intenta hacer frente al fenómeno de la violencia escolar se circunscriben al ámbito de lo legal o de la seguridad y en menor medida a la prevención. Poy (2014) reporta lo expuesto por Silvia Garza, quien aseguró que los marcos legislativos en México para prevenir y sancionar el acoso escolar no resuelven el problema, porque desconocen las prácticas normativas de las escuelas y no reconocen los aprendizajes adquiridos por las propias comunidades escolares. En opinión de Garza, la Ley para prevenir y atender el acoso escolar, bulliyng, tiene un carácter punitivo y enunciativo. Por tanto, plantea que la ley debe contemplar la erradicación de la violencia en todos los espacios donde viven los niños.

$\mathrm{Al}$ analizar la violencia escolar, Hernández (2014) plantea que las escuelas no están aisladas del resto de la sociedad; la fuente de esa violencia está fuera de las aulas no en su interior. Hernández considera que algo ha cambiado en las expresiones de violencia durante los últimos ańos, pareciera que se trata de mostrar que se es más que el otro, con más sańa y agresividad y menos escrúpulos que en el pasado.

Para Hernández, un factor que influye en este fenómeno es el desprestigio sistemático de los maestros promovido por la derecha empresarial, ya que esta campaña de desprestigio ha mellado su autoridad dentro del aula.

\section{Las múltiples dimensiones del fenómeno de la violencia escolar en la investigación educativa}

Aguilera, Muñoz y Orozco (2007) obtuvieron el índice de participación en actos de violencia, participación en peleas, robo, intimidación a compañeros, con base en la información de la 
encuesta sobre el contexto de los alumnos. En primaria es de $34.8 \%$ y en secundaria de $38.6 \%$. Estos autores reportan también el índice de victimización que se refiere a haber sido víctima de robo, burla, daño físico, miedo de ir a la escuela, que en primaria fue de $35.5 \%$ y en secundaria $39.4 \%$.

$\mathrm{El}$ índice de violencia fuera de la escuela, que se refiere a acciones que se presentan fuera de los establecimientos escolares: portación y uso de armas de fuego, robo con violencia, robo sin violencia, agresión física o verbal a alumnos y profesores y peleas; en primaria es de $60.9 \%$ y en secundaria de $60.7 \%$. El índice de violencia externa es claramente mayor que la interna de la escuela.

Aguilera, Muñoz y Orozco (ibid.) observaron que los hombres participan y son víctimas de violencia en mayor medida que las mujeres. En los varones son más evidentes y frecuentes las agresiones físicas y verbales así como el uso de lenguaje soez; en cambio, las mujeres utilizan, especialmente, otras formas de agresión verbal como la burla y algunas más sutiles, con un fuerte componente actitudinal (o de lenguaje no verbal), como la marginación. Por otra parte, los alumnos que presentan alguna condición personal desfavorable (como dificultad para desplazarse, ver, oír, etc.) manifiestan ser objeto de mayor agresión por parte de sus compañeros.

Los autores mencionados reportan que cuando estudiantes y profesores perciben que en su escuela hay exigencia académica y la disciplina es razonablemente estricta, las manifestaciones de violencia son menores. Sin embargo, cuando los alumnos perciben que la disciplina es muy exigente, hay un sensible aumento de conductas violentas y cuando la disciplina es extremadamente rígida y se aplica discrecionalmente, disminuye la efectividad del sistema disciplinario. El uso de sanciones es la forma de controlar las conductas violentas. Así mismo, estos investigadores encontraron que los maestros tienden a minimizar los problemas de convivencia entre estudiantes y que los alumnos perciben que no son atendidos cuando se enteran de ellos, particularmente acciones menos graves, como las burlas.

En cuanto a la composición y dinámica familiar, cuando los jóvenes viven en hogares monoparentales o donde los padres no están, el nivel de violencia se incrementa; lo mismo sucede 
cuando perciben que al interior de sus hogares la convivencia es conflictiva, y cuando los padres no están atentos a lo que hacen sus hijos fuera de la escuela.

Otra perspectiva la ofrecen Castillo y Pacheco (2008), quienes analizan que una manera de maltrato es la exclusión; $74.6 \%$ de los jóvenes observa que en sus escuelas hay compañeros a quienes se les ignora y se le deja solos, mientras que 59\% indicó que hay a quienes los propios jóvenes no les permiten participar en las actividades.

Otra cara del maltrato, según Castillo y Pacheco, es la violencia institucional que se hace presente cuando los estudiantes y familiares manifiestan sus quejas y no son escuchados por las autoridades educativas, que niegan el problema y de esta manera protegen a los agresores y exponen a sus víctimas, esta situación se replica en la familia.

Además de la violencia activa, las autoras identifican la violencia pasiva que asume la forma de indiferencia. Las faltas de respeto son vistas en la escuela como formas normales de socialización porque están veladamente permitidas en la institución. Los jóvenes están acostumbrados a recibir maltrato, frecuentemente prefieren que les digan apodos a ser ignorados. Las autoras plantean que la normalización y la valoración positiva de la violencia, como algo que hay que tolerar, puede ser indicador de un proceso de socialización en la violencia, lo cual disminuye la posibilidad de reconocerla.

Para Castillo y Pacheco (2008) los problemas de violencia no ocurren aislados de las relaciones sociales de los jóvenes, sino que están en sintonía con el ambiente social y cultural; las escuelas reproducen las relaciones de poder que le circundan: la ley del más fuerte, las faltas de respeto, los abusos, los malos tratos, los robos.

En el estudio realizado por Prieto (2005) se reportan hechos graves de violencia en escuelas secundarias, con formas tan sofisticadas de organización para el vandalismo, que ni los estudiantes, ni los docentes o directivos han podido enfrentar eficazmente. Prieto da un peso importante al papel del entorno en las experiencias de los alumnos, particularmente de la colonia o colonias que frecuentan; si en ellas hay cantinas, centros nocturnos, problemas de alcoholismo, drogadicción, en poco tiempo 
los jóvenes empiezan a reproducir las conductas que ven y oyen de su entorno. Para algunos de los alumnos de secundaria es la escuela donde por primera saben lo que es el miedo, el robo, las peleas, el consumo de alcohol y las drogas.

Pérez y Gálvez (2011) describen aspectos de lo que llaman violencia sistémica en las concepciones de alumnos y docentes y en las prácticas de docentes. Estas autoras se refieren a la violencia sistémica como cualquier práctica o procedimiento institucional que produzca cualquier efecto adverso en los individuos o en los grupos al imponerles una carga psicológica, mental, cultural, espiritual, económica o física y por tanto, califican como violencia sistémica aquellas prácticas y procedimientos que imposibiliten el aprendizaje de los alumnos.

Entre las prácticas escolares violentas se encuentran aquellas que estigmatizan a los alumnos; las autoras destacan, además, la estandarización, en cuanto a que se trabaja un único currículo para todos los alumnos, independientemente de su pertenencia cultural, intereses y necesidades. Las expectativas de los docentes operan en favor o en contra de los alumnos basadas en su apariencia y conducta. La organización de la escuela con un alto número de alumnos contribuye al alejamiento entre el docente y los estudiantes, lo mismo que la percepción de los maestros de que el objetivo de la escuela es únicamente cognitivo, lo cual separa la afectividad y otros aspectos que actúan en conjunto en la experiencia educativa. Finalmente, las investigadoras destacan que la institución ha normalizado el uso del castigo para disciplinar a los alumnos.

El artículo de Marina Camargo (1996) aporta una visión multidimensional de la violencia escolar en el contexto latinoamericano que permite amarrar algunos de los aspectos abordados por los investigadores educativos mencionados.

Camargo establece múltiples relaciones entre la violencia macro o social y la violencia escolar. La violencia social que se enfrentaba entonces en Colombia, se caracterizaba por los excesos en términos de violación a los derechos humanos, de desvalorización de la vida, una violencia visible y cotidiana.

Esta autora encuentra que no hay una relación directa entre la violencia social y la violencia escolar, pero que algunas 
concepciones, prácticas y relaciones que generan espacios de intolerancia, discriminación, exclusión, desconocimiento y aniquilación simbólica del otro en la escuela, dan lugar a semillas o brotes de violencia. A nivel político y social se siembra violencia cuando el Estado no cumple en términos de cobertura, retención y pertinencia de la educación.

En la escuela se generan semillas de violencia en la relación entre el maestro y el alumno mediante el señalamiento de rasgos, vacíos, deficiencias y defectos que no se ven como algo a superar sino como "taras" que cargar. Las correcciones son poco formativas, se recurre a la ridiculización frente a compañeros, humillaciones y ofensas al alumno. El regaño es una forma de explicar, evaluar, corregir o reprender por la contravención disciplinaria o académica.

La inadecuación o ausencia de mecanismos de justicia capaces de resolver conflictos en la escuela genera condiciones favorables para la violencia, como el maltrato o los castigos. También contribuyen a ello las maneras de abordar el conocimiento de forma verticalista y autoritaria, ignorante del saber del alumno y, por tanto, sin sentido para este.

\section{Algunos rasgos de los contextos y las violencias en escuelas de educación básica en Morelos, México}

La estructura de presentación de las narraciones de los docentes se hará siguiendo las características de la situación de guerra expuesta en este documento por Ceceña (2014): avasallamiento, simultaneidad e impunidad.

A continuación se presentan fragmentos de las narraciones de docentes que dan cuenta de la manera en la que la violencia se está viviendo en los contextos comunitarios, escolares y familiares.

En cuanto a situaciones de avasallamiento en las que están presentes la desigualdad, la pobreza, el hambre y la violencia:

La escuela para los más desprotegidos es considerada como una oportunidad para mejorar su calidad de vida, es una esperanza para salir de su medio de pobreza y hambre, pero qué pasa cuando no se les brinda lo que ellos aspiran y en lugar de ello, sufren violencia o agresión. Los docentes 
o padres de familia lo perciben como normal, ignoran las afectaciones emocionales y psicológicas, que la violencia pudiera tener (LC-1-05-14).

Los padres tienen subempleos, pepenadores de basura, maquiladores, peones, de ellos pocos obtienen sueldos fijos, muchos son temporales, las madres se dedican a realizar aseos en casas ajenas, su nivel de estudios es de primaria. Por las mañanas los alumnos asisten con solo café y pan, si es que sus mamás se preocupan, si no, asisten con el estómago vacío (LC-11-05-14).

La pobreza con la que viven los alumnos es tanta, que se han reportado casos de robo de dinero y objetos dentro de los salones de clases, por hambre (DMO-11-05-14).

Así manifiestan los docentes la experiencia de la pobreza, las tensiones en la familia y el maltrato:

Muchos padres de familia, al no encontrar empleos dignos, tienen que comprometerse a laborar largas horas al día en trabajos informales donde no se les brinda ni lo más mínimo en prestaciones laborales que solventen la seguridad de la familia; por tal motivo, se ven en la necesidad de dejar a "la suerte" la integridad física y emocional de sus hijos (DMO-8-05-14).

Las formas en que los niños se relacionan, reciben y manifiestan la violencia es alarmante; en ocasiones muestran a sus maestros huellas de golpes en cierta parte de su cuerpo, algunas veces dejan entrever o relatan la forma en que se les aplican correctivos a través de palabras altisonantes o golpes por parte de sus mentores, hermanos o parientes (DMO-10-05-14).

Situaciones de conflicto en las comunidades que afectan las relaciones entre alumnos

La gran mayoría de personas que ahora vive de manera irregular en esta colonia, fue por la necesidad de tener una vivienda, aunque lamentablemente son terrenos comunales de la reserva del Texcal y continuamente están en conflictos legales. Los pobladores llegan en calidad de paracaidistas, invadiendo terrenos comunales; comentan algunas personas de la 
comunidad que, por el simple hecho de provenir de Guerrero, los etiquetaban como "ladrones" (sg-10-05-14).

La Colonia se encuentra muy fragmentada tanto por la religión como por los partidos políticos y las rencillas entre familias (LC-9-05-14).

Experiencias de simultaneidad: exclusión, discriminación, represión, condiciones adversas para el aprendizaje en la escuela

$\mathrm{Al}$ alumno se lo ubica en un lugar, por imposición más que por gusto y en algunos casos se le designa el lugar por sus características intelectuales, económicas, físicas, conductuales, entre otras, y esto propicia que, desde el primer día de clases, el niño ya esté etiquetado (sg-2-05-14).

Violencia como discriminación entre pares y deterioro del ambiente escolar

La violencia entre pares se da cuando unos alumnos les dicen a otros que no se "junten" con alguno de sus compañeros, que no dejen que hagan equipo con ellos o se reúnan en la hora del recreo para jugar o compartir el lunch (DMO-13-05-14).

Para ejemplificar los casos que se viven diariamente, comparto un caso dentro de la escuela:

Perla, de 6 años, es una niña que en su apariencia física está muy descuida$\mathrm{da}$, tiene piojos y aunque sus vecinas la limpien constantemente, este problema vuelve a aparecer; su ropa la lleva completamente sucia despidiendo un olor muy desagradable, tiene la mayoría de sus dientes con caries. Me di cuenta que su mamá trabaja fuera de su casa, pero nadie sabe en qué. Perla y sus tres hermanos están al cuidado de sus vecinos, a veces comen $\mathrm{y}$ a veces no, $\mathrm{y}$ al parecer su hermano de cuarto grado fue violado por un vecino. Sus compañeros rechazan completamente a Perla y ella es muy agresiva, si alguien se le queda viendo "feo", así lo ha dicho ella, va hasta su lugar y les jala el cabello o les tira sus útiles y se justifica diciendo que la están molestando y por ello reacciona así (sg-11-05-14).

Unión de bajas expectativas escolares y permiso para agredir 
La principal preocupación de los padres o tutores es que haya un lugar donde cuiden a sus hijos en las horas en que ellos trabajan, no tienen tanto interés en que su proceso de aprendizaje sea idóneo (sg-5-05-14).

He escuchado comentarios como: "obedece a tu maestra", "si te pegan, tú también pégales", "cuida a tu hermanito de que no le hagan nada", "si llegas golpeado a la casa, ya verás cómo te va", "no quiero ver que te juntes con esa nińa sucia”. Considero que esto genera un conflicto en el nińo entre obedecer a sus padres, que son los que emocionalmente están más cercanos y a los que les tiene un cierto respeto (sg-6-05-14).

Represión como forma de disciplina y negación de la individualidad

Desde que el niño ingresa al espacio escolar, ya lo hace con la consigna de que "no debe de platicar", "no debe de jugar", "no debe de levantarse de su lugar" (sg-1-05-14).

Se clasifica a los alumnos en categorías duales, entre los buenos y malos, disciplinados e indisciplinados, a partir de la idea de un alumno modelo, representación que se nos ha formado de un niño que cumple con exactitud el modelo, el que tiene más capacidad para memorizar, el más obediente y disciplinado, el más eficiente para los trabajos (sg-8-05-14).

\section{Docentes presionados, maltratos y que maltratan}

Es importante reconocer que en las instituciones educativas, también los maestros son o han sido víctimas de violencia escolar, desde el momento en el que burocráticamente se les exige cumplan con la normatividad impuesta por las autoridades educativas, como lo son las funciones administrativas, "el papeleo", además de las cuestiones pedagógicas, obligándolos a abandonar a los grupos en horarios de clases para rendir informes de distinta índole y, en caso de incumplir, se les sanciona administrativa o económicamente. A los maestros también se les amenaza con separarlos de sus cargos si no aprueban evaluaciones de conocimientos (DMO-3-05-14).

Hace unos años, la escuela se vio envuelta en una problemática fuerte, debido a que un grupo de padres demanó ante derechos humanos a la 
profesora de un grupo de tercer grado, argumentando que sus hijos eran agredidos física y verbalmente por la responsable del grupo. También se habló de que la maestra los golpeaba cuando la hacían enojar (DMO-13-05-14).

\section{Narración de las condiciones escolares adversas, intolerancia y agresión}

La institución educativa en la cual laboro está ubicada en un contexto urbano de marginalidad y de vulnerabilidad; al ser la única en un total de cuatro colonias de nueva creación, la cantidad de alumnos influye directamente en la violencia escolar al no haber espacio suficiente. La escuela tiene los grupos saturados, con un número de alumnos que oscila entre los 37 y los 59. Esto dificulta el trabajo del maestro y no se puede proporcionar una atención adecuada a los alumnos con problemas que requieran atención especial, ya sea como agresor o como agredido (sg-13-05-14).

Las interacciones que se dan entre los alumnos del primer ciclo de la escuela primaria en la localidad El Pedregal, Ocoyoacac, México, hacen patente situaciones conflictivas que llaman la atención. Entre los conflictos que se han identificado en el grupo de primer grado, están las situaciones de inconformidad ante las tareas de equipo, algunos alumnos rehúyen a esta dinámica de trabajo porque manifiestan sentirse incómodos, porque ante los niños o niñas no les gusta colaborar, entonces se oyen frases como "es una burra", "es que no sabe leer" o "no les gusta hacer nada"; quienes manifiestan inconformidad, son niños o niñas que están más avanzados en su proceso de adquisición de la lecto-escritura (Po-1-05-14).

\section{Experiencias de impunidad}

El contexto social se ha vuelto más hostil, y se percibe miedo e incertidumbre entre los habitantes y trabajadores. Algunos maestros, padres de familia y alumnos que asisten a la escuela primaria han sido víctimas de asaltos a mano armada, agresiones verbales y extorsión; en una calle aledańa a la Primaria, hubo intento de homicidio a una maestra que, al pretender asaltarla, le dispararon en dos ocasiones; los vecinos, padres y alumnos de la institución fueron testigos de esto. Las autoridades también son víctimas de violencia, por ejemplo a una directora del turno matutino, la 
abofetearon a las afueras de la escuela, por no haber inscrito a un menor (DMO-16-05-14).

Situaciones de impunidad en la escuela

Algunos niños logran infundir miedo, angustia a otros al obligarlos a realizar cosas en contra de su voluntad. En una ocasión, por ejemplo, unos alumnos (varones) de primer grado obligaron a uno de sus compañeros a realizarles sexo oral, amenazándolo que de no hacerlo lo iban a golpear (DMO-14-05-14).

Niños y niñas sin sueños de porvenir

Durante la intervención educativa que realicé con alumnos de primer y sexto grados, observé que todos los chicos de primero, sin importar su condición socioeconómica, soñaban en cómo sería su porvenir y querían realizar grandes cosas. Algunos soñaban con ser médicos, pilotos de avión, maestras, enfermeras, futbolistas, músicos, cantantes policías y bomberos. Sin embargo, en sexto no sucedía lo mismo. Al ser interrogados al respecto, manifestaron que "esas eran tonterías de escuincles" y que ellos, al haber crecido y madurado, se consideraban mucho más prácticos. Ya no soñaban, sino que buscaban lo "real”, lo factible para ganar dinero rápido y resolver así su futuro... les parecía mejor buscar "chamba" para ayudar en su casa. Muchas nińas de trece o catorce años, pensaban que una buena solución sería "juntarse" con un "chavo de varo" que las mantuviera. Ninguno se sentía capaz de ir a la universidad "solo los niños fresas van ahi”" (FB-1-05-14).

\section{CONCLUSIONES}

Las características del tipo de guerra mencionados antes: avasallamiento, simultaneidad e impunidad se articulan con elementos de análisis propuestos por Foucault: saber, poder y subjetividad que forman una red, un entramado de relaciones sociales que, al analizarlo, puede dar luz sobre formas de violencia que se han normalizado y que por tanto permanecen invisibles.

Lo que se quiere conocer a partir de estos elementos son las relaciones sociales, el lugar del sujeto y las relaciones de poder $\mathrm{y}$ 
saber implícitas o explícitas en la narración. Al respecto, Foucault dice que son "...prácticas que configuran sistemáticamente los objetos de los que hablan... Los discursos no se refieren a objetos; no identifican objetos; los construyen y, al hacerlo, ocultan su propia invención" (1970: 49).

Las relaciones de poder, saber y subjetivación que narran los docentes al expresar su experiencia en la vida cotidiana de las comunidades, la familia y la escuela se caracterizan como violentas en el sentido de que, como señala Maldonado (2004: 5), violencia es toda situación de poder que naturalice la desigualdad y la jerarquía, que transforma a sujetos en objetos. En las narraciones presentadas en esta investigación se encuentran situaciones de exclusión y discriminación e impunidad en todos los ámbitos sociales, empezando por el Estado al incrementar la desigualdad y abandonar su papel como regulador de las condiciones de vida de la población y su seguridad.

Las relaciones sociales en los contextos descritos se caracterizan también por el autoritarismo y las jerarquías. Las relaciones de poder ligadas a la obtención de bienes materiales o prestigio en los ámbitos comunitario, escolar, familiar, eclipsan los esfuerzos de los distintos actores por centrar su atención en el aprendizaje y destruyen la cohesión. En ese tipo de relaciones el sujeto es minimizado o convertido en objeto.

Con base en la teoría, experiencia y visiones, por un lado, de expertos $y$, por otro, de actores que viven la violencia de manera directa abordados en este estudio, puede decirse que entre la violencia social y la violencia escolar hay una dinámica compleja, algunos puntos de articulación pueden reconstruirse de esta manera: por las condiciones de desigualdad y de guerra en nuestro país, llegan a la escuela niñas, niños y jóvenes despojados de sus derechos humanos, sociales y políticos, que viven en condiciones de precariedad, escasez, desesperanza, desamparo y desilusión, control no legitimado de los territorios, violencia desbordada; al mismo tiempo, el orden social imperante, contradictorio y con múltiples hilos entre lo legal e ilegal, actúa como ambiente sociomoral en el que se socializan niñas, niños y jóvenes mexicanos quienes interactúan, observan, escuchan y están en contacto con ideas y prácticas que cuestionan y ponen en tensión los valores 
sociales, culturales y simbólicos; estos se dan cuenta de la explotación laboral, de la desigualdad, las jerarquías paralelas, de los negocios inmorales, los conflictos familiares, vecinales, los dobles discursos, la impunidad, la inequidad, la complicidad, la desvalorización de la vida humana y temen o sufren el desbordamiento de la violencia en sus comunidades.

Poniendo en uso las palabras de Ceceña, niñas, niños y jóvenes experimentan en su cotidianidad, por todos lados y al mismo tiempo, el despojo, el saqueo, el abuso de poder, el uso de la fuerza para dirimir las diferencias, el transcurrir de una vida en tierra de nadie. Estas situaciones objetivas se acompañan de aspectos subjetivos como el predominio del miedo, la desconfianza en los otros, la incertidumbre y la desesperanza.

La situación descrita constituye lo que Madriaza (op. cit.) llama un fondo social del cual emerge la violencia entre pares, en la escuela y fuera de ella, como un síntoma del deterioro social. El sistema educativo y la escuela forman parte de ese fondo social que engendra violencia, cuando fomenta la normalización de la exclusión, el autoritarismo, el castigo como disciplina, la arbitrariedad de las normas, las bajas expectativas, el maltrato, la desconfianza hacia los docentes y cuando se vulnera su autoridad, el exceso de carga administrativa, grupos muy numerosos, condiciones precarias de las instalaciones educativas. Además, la violencia se favorece con la ruptura entre los contenidos rígidos, las formas de enseñanza, y las necesidades, intereses, características personales y medio cultural de los alumnos, entre los aspectos cognitivos y los socioafectivos; entre lo que los niños son y quieren ser y lo que el sistema educativo quiere que sean.

La escuela llega a hacer una contribución a la violencia social cuando no evita que en la socialización cotidiana en la institución se den situaciones que propician, en palabras de Camargo (1996), el aprendizaje de hacerse justicia por la propia mano, de aniquilar al diferente, de usar la fuerza como instrumento para resolver conflictos. De no atenderse la forma de socialización en todos los ámbitos sociales se aniquilan y se afectan, de manera negativa, los proyectos de vida, las maneras de vivir, las visiones de sociedad de niñas, niños y jóvenes. 


\section{REFERENCIAS BIBLIOGRÁFICAS}

Aguilera, Ma. Antonieta, Gustavo Muñoz, Adriana Orozco, Disciplina, violencia y consumo de sustancias nocivas a la salud en escuelas primarias y secundarias de México, México, Instituto Nacional para la Evaluación de la Educación (INEE), 2007.

Blanco, Mercedes. "Investigación narrativa: una forma de generación de conocimientos", en Argumentos, 24, septiembre-diciembre, 2011. Disponible en:<http://redalyc.org/ articulo.oa?id=59521370007 $>$ Fecha de consulta, 11 de junio de 2014.

Bourbaki. El costo humano de la guerra por la construcción del monopolio del narcotráfico en México (2008-2009), México, Equipo Bourbaki, 2011.

Bourdieu, Pierre. Razones prácticas. Sobre la teoría de la acción, 2a edición, Barcelona, Anagrama, 1999.

Camacho, Fernando. "Alerta relator de ONU sobre alarmante nivel de violaciones al derecho a la vida", en periódico $L a$ Jornada, 11 de junio de 2014.

Camargo, Marina. "Violencia escolar y violencia social", 1996. Disponible en http://www.acosomoral.org/pdf/ rce34_03ensa.pdf. Fecha de consulta, 20 de marzo de 2010. Castillo, Carmen y María Magdalena Pacheco. "Perfil del maltrato (Bullying) entre estudiantes de Secundaria en la Ciudad de Mérida, Yucatán”, en RMIE, vol. 13, núm. 38 julio-septiembre 2008, pp. 825-842.

Ceceńa, Ana E. "Los golpes de espectro completo", en Revista América Latina en Movimiento, núm. 495, mayo de 2014.

CEPAL. "Panorama Social de América Latina y el Caribe. Documento informativo, 2013". Disponible en http:// www.asocamerlat.org/CEPAL_PanoramaSocial2013_ AmericaLatina_diciembre2013.pdf. Fecha de consulta, 11 de junio de 2014.

Colombo, Jorge. "Acerca del desarrollo cerebral infantil: entre el daño y la optimización social”, en Colombo, Jorge (Ed.) Pobreza y desarrollo infantil. Una contribución multidisciplinaria, Buenos Aires, Paidós, 2007. 
Foucault, Michel. La arqueología del saber, México, Siglo XXI, 1970. García, Rosario y Roberto González. "La gestión de la violencia en las escuelas secundarias de Iztapalapa", en Memoria del XI Congreso Nacional de Investigación Educativa, México, COMIE, 2011.

Garduño, Roberto y Enrique Méndez. "México quinto lugar mundial en delincuencia”, en periódico La Jornada, 24 de enero de 2009.

González, Susana. "Limitado el impacto laboral de Oportunidades: CEPAL y OIT", en periódico La Jornada, 2 de junio de 2014.

Hernández, Luis. "El escándalo del acoso escolar", en periódico La Jornada, 10 de junio de 2014.

Kruijt, Dirk. "Violencia y pobreza en América Latina: los actores armados", en Revista Pensamiento Iberoamericano, núm. 2, 2a época, 2008, pp. 55-70.

Lieblich, A., R. Tuval-Mashiach y T. Zilber, Narrative research, Londres, Sage, 1998.

López, Lara y Antonia Ramírez. "Medidas disciplinarias en los centros educativos, ¿suficientes contra el acoso escolar?", en Perfiles Educativos, vol. XXXVI, núm. 145, México, IISUE-UNAM, 2014, pp. 32-50.

Madriaza, Pablo. Sentido social de la violencia escolar, Tesis de grado, Chile, Universidad de Chile, 2006. Disponible en http://www.tesis.uchile.cl/tesis/uchile/2006/madriaza_p/ sources/madriaza_p.pdf. Fecha de consulta, 9 de junio de 2014.

Maldonado, Stella. Prólogo del libro La Escuela como territorio de intervención política, Argentina, CTERA, 2004.

Márquez, David. "Bullying económico. Pobreza en la infancia y la adolescencia", en periódico La Jornada, 23 de junio de 2014.

Mèlich, Joan-Carles. Filosofía de la finitud, Barcelona, Herder, 2002.

Moreno, Florentino. Violencia colectiva, violencia politica, violencia social, aproximaciones conceptuales, s. 1., 2006. Disponible en http://documentacion.aen.es/pdf/libros-aen/coleccionestudios/violencia-y-salud-mental/parte1-algunos-conceptos-sobre-la-violencia/019-aproximaciones-conceptuales. pdf. Fecha de consulta, 10 de marzo de 2013. 
O’Donnell Porto, Anahí. "Las carencias alimentarias en el país. $\mathrm{Su}$ impacto sobre el desarrollo infantil", en Colombo, Jorge (Ed.), Pobreza y desarrollo infantil. Una contribución multidisciplinaria, Buenos Aires, Paidós, 2007.

OCDE/TALIS. Segundo Estudio Internacional sobre la Enseñanza y el Aprendizaje, 2013. Disponible en https://www.oecd. org/edu/school/Mexico-TALIS-2013_es.pdf. Fecha de consulta, 11 de marzo de 2014.

Pérez, Maritza y Marlén Gálvez. "La violencia sistémica en la práctica educativa, un fenómeno visto desde los alumnos y los docentes", en Memoria del XI Congreso Nacional de Investigación Educativa, México, COMIE, 2011.

Poy, Laura. "Marcos legislativos aplicados en México no resuelven el problema del acoso escolar", en periódico $L a$ Jornada, 27 de junio de 2014.

Prieto, Patricia. "Violencia escolar y vida cotidiana en la escuela secundaria”, en RMIE, vol. 10, núm. 2, oct-dic ,2005, pp. 1005-1026.

Rodríguez, Gabriela "Los Olvidados", en periódico La Jornada, 30 de mayo de 2014.

Santos, Boaventura. Descolonizar el saber, reinventar el poder, Uruguay, Ediciones Trilce, 2010.

Yurén, Ma. Teresa. Materiales de trabajo de la especialidad en herramientas básicas para la investigación educativa, México, Instituto de Ciencias de la Educación, Universidad Autónoma del Estado de Morelos, 2011.

Zemelman, Hugo. Conocimiento y sujetos sociales. Contribución al estudio del presente, México, El Colegio de México, 1987. 